\title{
Measuring entanglement using quantum quenches
}

\author{
John Cardy \\ Rudolf Peierls Centre for Theoretical Physics, Oxford University, \\ 1 Keble Road, Oxford, OX1 3NP, United Kingdom \\ All Souls College, Oxford, United Kingdom and \\ Kavli Institute for Theoretical Physics, Santa Barbara
}

(Dated: March 7 2011)

\begin{abstract}
We show that block entanglement entropies in one-dimensional systems close to a quantum critical point can in principle be measured in terms of the population of low-lying energy levels following a certain type of local quantum quench.
\end{abstract}

Entanglement, and its quantification through entanglement entropy, have become increasingly important tools in the study of quantum many-body systems. The fact that the entanglement entropy of a region of linear size $\ell$ in the ground state of a system with short-range interactions in $d$ dimensions grows like $\ell^{d-1}$ [1] (up to possible logarithms), as compared with a typical state where it is extensive, both explains the success of modern numerical methods and informs their further development. Moreover it gives a basis-independent way of detecting quantum critical behavior and topological phases.

Briefly, the entanglement entropy is defined as follows: given a bipartition of the Hilbert space $\mathcal{H}=$ $\mathcal{H}_{A} \otimes \mathcal{H}_{B}$ (which usually corresponds to the degrees of freedom lying in mutually exclusive regions $A$ and $B$ of $d$-dimensional space), the reduced density matrix of (say) $A$ is given by $\rho_{A}=\operatorname{Tr}_{\mathcal{H}_{B}}|0\rangle\langle 0|$. The Rényi entropies are then given by $S_{A}^{(n)}=(1-n)^{-1} \log \operatorname{Tr}_{\mathcal{H}_{A}} \rho_{A}^{n}$. The von Neumann entropy $-\operatorname{Tr}_{\mathcal{H}_{A}} \rho_{A} \log \rho_{A}$ is formally the limit as $n \rightarrow 1$. For different $n$ these encode the entanglement spectrum of $\rho_{A}$ : if we make a Schmidt decomposition

$$
|0\rangle=\sum_{k} c_{k}|k\rangle_{A}|k\rangle_{B}
$$

then the non-zero eigenvalues of $\rho_{A}$ are given by $c_{k}^{2}$. On the other hand $\operatorname{Tr}_{\mathcal{H}_{A}} \rho_{A}^{n}=\sum_{k} c_{k}^{2 n}$. If the state has low entanglement then only a few eigenvalues are appreciable and the entropies are small, while if the state is maximally entangled the entropies are of the order of the lesser of the dimensions of $\mathcal{H}_{A}$ and $\mathcal{H}_{B}$.

In exactly solvable models, and more generally for systems at or near a quantum critical point, considerable progress has been made in relating the behavior of the entanglement entropy to other universal data of the underlying long-distance theory, typically a quantum field theory (QFT) [2]. In particular, for $d=1$, the coefficient of the 'area' law (in this case the number of boundary points between $A$ and $B$ ) is predicted to diverge as $\log \xi$, where $\xi$ is the correlation length, as the critical point is approached. At the critical point, the entropy of a finite interval of length $\ell$ immersed in a much larger system grows like $\log \ell$. When the low energy, long wavelength physics is described by a conformal field theory (CFT), the coefficient is given by the central charge, or conformal anomaly number, $c$ : the Rényi entropies behave as [3, 4] $S_{A}^{(n)} \sim(c / 6)\left(1+n^{-1}\right) \log \ell$. Many other predictions have been made by now. For example, when $A$ consists of two disjoint intervals, the entropy encodes all the data of the CFT, which is therefore recoverable solely from properties of the ground state wave function $[\underline{5},[6]$. These predictions, both in $d=1$ and higher, have been well verified in numerical investigations, and indeed the low degree of entanglement is partly responsible for the success of DMRG and tensor network methods [7].

However it seems difficult to devise a method whereby entanglement entropy in an extended system of the type discussed above could be measured, even in principle, in a real experiment, since it is intrinsically non-local. While various methods have been proposed for measuring entanglement in systems with a finite dimensional Hilbert space [8], their complexity increases with the system size. What we have in mind is a conventional condensed matter experiment whose difficulty does not in principle increase with the system size. In some simple systems the entanglement entropy can be indirectly recovered from an (in principle) measurable correlation function 9, 10] or the distribution of suitably chosen observables [11], but this connection is system-specific.

Various suggestions have been made as to how charge [12, 13] or number [14] fluctuations in the subsystem $A$, either in space or in time, may provide a measurement of entanglement entropy. However, the generality of these observations has been questioned [15]. They are restricted to systems with a conserved current, and it is difficult to see how these ideas could measure entanglement of neutral degrees of freedom, or apply in cases when there is no such conservation law. More seriously, the logarithmic behavior which appears in these analyses can be traced to the fact that, in one dimension, currentcurrent correlators $\left\langle J\left(x_{1}\right) J\left(x_{2}\right)\right\rangle$ behave like $\left|x_{1}-x_{2}\right|^{-2}$ on separations much smaller than the size of the subsystem (but larger than the microscopic scale, and similarly in the time domain), giving rise to logarithms on integration over $x_{1}$ and $x_{2}$. Although the functional form of such logarithms turns out to be identical to that of the entanglement entropy for simple geometries, the analysis 
of Ref. 6 shows that, even in the slightly more complicated case where $A$ consists of two disjoint intervals, the entanglement entropy has a form which cannot be simply expressed as an integral over correlation functions of local fields.

The proposal in Ref. 12 is an example of a local quantum quench 16, 17], whereby the hamiltonian of a quantum system is instantaneously changed $H \rightarrow H^{\prime}$ in a local way, so that the quantum state, which was the ground state of $H$, now evolves according to $H^{\prime}$. In general, the real time behavior after such a local quench is relatively simple [17]: the additional energy near the quench site is radiated away as the quasiparticles of $H^{\prime}$, moving semiclassically at their group velocities $v_{g}$. These also propagate changes in entanglement through the system, which, in a critical $1 \mathrm{~d}$ system, therefore grow like $\log \left(v_{g} t\right)$.

In this letter we propose a different type of local quantum quench which, in principle, directly measures the Rényi entropies. We show that these are simply given by the probability $P_{0}=\left.\left.\right|_{H^{\prime}}\langle 0 \mid 0\rangle_{H}\right|^{2}$ of finding the system in the ground state of $H^{\prime}$ after the quench. This quantity is called the fidelity, and it has been used extensively in characterizing quantum critical behavior (see, for example, Ref. 18), but it has largely been restricted to global, rather than local changes in the hamiltonian. (See, however, [19].) It may be objected that $P_{0}$ is not directly experimentally observable, even in a gapped system. However we also show that, in systems close to a quantum critical point described by a QFT with a linear dispersion relation, the probability $P(E)$ of finding the system in a low-lying excited state of energy $E$ is given by $P_{0}$ times a calculable factor. In principle, $P(E)$ is measurable if the system is coupled weakly in a known fashion to other modes whose spectrum can be analyzed. This then gives an indirect measurement of $P_{0}$ and hence the Rényi entropy. The analog of $P(E)$ for global quenches has been discussed in [20, 21].

Let us define more precisely the local quench. In computing Rényi entropies, it is convenient to imagine $n$ copies of the original system. We now suppose that these copies can actually be manufactured, to a tolerance to be discussed later. Consider therefore $n$ identical copies of the system so that the full Hilbert space is $\mathcal{H}=\otimes_{j=1}^{n} \mathcal{H}_{j}$. Initially they are decoupled so that the hamiltonian is $H=\sum_{j=1}^{n} H_{j}$, and each system is in its ground state (assumed unique), so the combined system is in its ground state $|0\rangle_{H}=\prod_{j}|0\rangle_{j}$. Consider the same bipartite decomposition of each $\mathcal{H}_{j}=\mathcal{H}_{j A} \otimes \mathcal{H}_{j B}$. All the $\mathcal{H}_{j A}$ are isomorphic. Let $\Pi_{n}$ be the permutation operator (unitary on $\mathcal{H})$ which maps $\mathcal{H}_{j A} \rightarrow \mathcal{H}_{(j+1) A}(\bmod n)$ and acts as the identity on all the $\mathcal{H}_{j B}$. (Such an operator for the case $n=2$ was introduced in Ref. 22 where it is called a swap operator.) Then it is well-known (see e.g. Ref. 23 for the case $n=2$ ) that using the decomposition

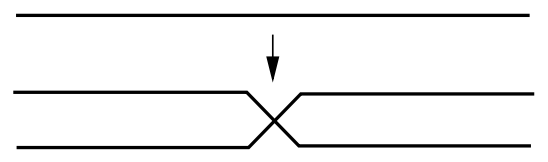

FIG. 1: The action of a single twist operator on two onedimensional systems with short-range interactions.

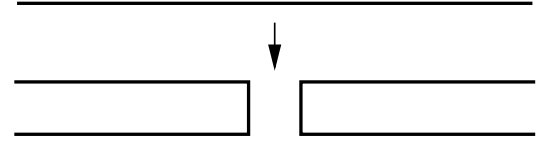

FIG. 2: A single twist is equivalent to the closing of a point contact when $n$ is even and the ground state is invariant under reflection.

(11) and the orthonormality of the Schmidt states,

$$
{ }_{H}\left\langle 0\left|\Pi_{n}\right| 0\right\rangle_{H}=\sum_{k} c_{k}^{2 n}=\operatorname{Tr}_{\mathcal{H}_{A}} \rho_{A}^{n}
$$

On the other hand, if we define $H^{\prime}=\Pi_{n}^{-1} H \Pi_{n}, H^{\prime}$ and $H$ are isospectral, and $|0\rangle_{H^{\prime}}=\Pi_{n}^{-1}|0\rangle_{H}$ is the ground state of $H^{\prime}$. Hence, thinking of $H \rightarrow H^{\prime}$ as a quantum quench, the modulus squared of (2) is nothing but $P_{0}=$ $\left|H^{\prime}\langle 0 \mid 0\rangle_{H}\right|^{2}$.

The point about this elementary observation is that, when $H$ has only short-ranged interactions, and $A$ and $B$ are spatially disjoint regions, the difference between $H$ and $H^{\prime}$ is restricted to the boundary between $A$ and $B$. Thus while the action of $\Pi_{n}$ on states, expressed in particular basis, may be complicated (see, e.g. Fig. 1 of Ref. 22), its action on the combined hamiltonian is simple. As a example, consider two copies of a Heisenberg spin chain with nearest neighbor interactions and hamiltonian $J \sum_{l} \sigma_{l} \cdot \sigma_{l+1}$. Take $A$ to be the set of sites with $l \leq 0$ and $B$ those with $l \geq 1$. Then

$H^{\prime}-H=J \sigma_{0}^{(1)} \cdot \sigma_{1}^{(2)}+J \sigma_{0}^{(2)} \cdot \sigma_{1}^{(1)}-J \sigma_{0}^{(1)} \cdot \sigma_{1}^{(1)}-J \sigma_{0}^{(2)} \cdot \sigma_{1}^{(2)}$

This is illustrated in Fig. 1 (For an odd number of such twists it is also necessary to twist the boundary conditions at the same time. This has no effect in open systems or for periodic boundary conditions in systems much larger than the correlation length, but is important for finite ungapped systems with periodic boundary conditions.) Such twist operators have been studied for integrable spin chains in 24].

Note that for an infinite system or one with periodic boundary conditions, one of the chains can be parity reversed as in Fig. 2 (assuming the ground state $|0\rangle$ is invariant under this reflection) to obtain a local quench corresponding to the closing of a point contact coupling to two external leads on either side. In a real experiment, 
of course, it may be difficult to ensure that the new couplings added in $H^{\prime}$ are precisely the same as those deleted in $H$. However, at least in a gapped system, the results should be robust to such imperfections as long as they are on scales smaller than the gap, or the energy $E$ of excited states.

The operators $\Pi_{n}$ corresponding to a local modification of $H$ at some point $l$ in a one dimensional system are called twist operators $\mathcal{T}_{n}(l)$ in the literature [25]. From the above we see that the Rényi entanglement entropy between the left and right halves of such a system is given in terms of the ground state expectation value ${ }_{H}\left\langle 0\left|\mathcal{T}_{n}(0)\right| 0\right\rangle_{H}$. Similarly the Rényi entanglement entropy between an interval of length $\ell=\left|l_{1}-l_{2}\right|$ and the rest of the system is given by ${ }_{H}\left\langle 0\left|\mathcal{T}_{n}\left(l_{1}\right) \mathcal{T}_{n}\left(l_{2}\right)\right| 0\right\rangle_{H}$, and so on. In all cases, these matrix elements may be viewed equivalently as the fidelity following a quantum quench of $H$ to $H^{\prime}=\prod_{k} \mathcal{T}_{n}\left(l_{k}\right)^{\dagger} H \prod_{k} \mathcal{T}_{n}\left(l_{k}\right)$, or equivalently the probability $P_{0}$ that the system is found in the ground state of $H^{\prime}$ when its energy is measured.

In one-dimensional systems close to a critical point, considerable effort has gone into analyzing the behavior of correlators of these twist operators, especially for systems with dynamical scaling exponent $z=1$, which are described in the scaling limit by a relativistic QFT, and at the critical point, by a CFT 2]. In these cases, it follows from the early work of Ref. 3 that $\mathcal{T}_{n}$ has scaling dimension $x_{n}=(c / 12)(n-1 / n)$, where $c$ is the conformal anomaly number of the CFT. By scaling, this means for a single twist in a system with a finite gap $\Delta, P_{0}$ scales as $b_{n}^{\prime} \Delta^{2 x_{n}}$ as $\Delta \rightarrow 0$. Similarly, for an interval of length $\ell$ at the critical points, it behaves like $b_{n} \ell^{-2 x_{n}}$. The constants $b_{n}$ and $b_{n}^{\prime}$ are not universal, but their ratio is expected to be, in units where $v=1$ [28].

However, measuring the total energy of a many-body system to accuracy $O(1)$ is unfeasible even when the spectrum is gapped. In fact, after such a local quench, most of the excess energy goes into states whose energy is of the order of the inverse cut-off, or band width. This can be seen by studying the time dependence of the energy density, given by the component $T_{00}(x, t)$ of the energy momentum tensor, following the action of a single twist. This is given in the Schrödinger picture by ${ }_{H}\left\langle 0\left|\mathcal{T}_{n}(0)^{\dagger} e^{i H t} T_{00}(x) e^{-i H t} \mathcal{T}_{n}(0)\right| 0\right\rangle_{H}$. On the other hand, in a CFT, we can write $T_{00}=T+\bar{T}$, and, in imaginary time

$$
\begin{aligned}
& { }_{H}\left\langle 0\left|\mathcal{T}_{n}(0)^{\dagger} e^{-H \tau^{\prime}} T(x) e^{-H \tau} \mathcal{T}_{n}(0)\right| 0\right\rangle_{H} \\
& =\frac{(c / 24)\left(1-n^{-2}\right)\left(\tau+\tau^{\prime}\right)^{2}}{(x+i \tau)^{2}\left(x-i \tau^{\prime}\right)^{2}}
\end{aligned}
$$

This follows from Eq. (11) of [4]. A similar result holds for $\langle\bar{T}\rangle$. Continuing this result to $\tau=i t+\delta$ and $\tau^{\prime}=-i t+\delta$, where $\delta$ is a UV cut-off of the order of the lattice spacing or inverse band width, we see that, after the quench,

$$
\left\langle T_{00}(x, t)\right\rangle \sim \frac{c}{12}\left(n-n^{-1}\right)\left(\frac{\delta^{2}}{\left((t+x)^{2}+\delta^{2}\right)^{2}}+\{x \rightarrow-x\}\right)
$$

in units where $v=1$. The total energy $\int\left\langle T_{00}\right\rangle d x$ diverges like $\delta^{-1}$, and, in a CFT, it is concentrated in a region of width $O(\delta)$ near the light cone. In a more general lattice theory we expect the energy to be carried off by quasiparticles moving semi-classically with their appropriate group velocities. Similar considerations apply to changes in the entanglement entropy [17].

We now argue that, despite the fact that most of the energy is radiated in this non-universal manner, the population $P(E)$ of the states with energies $E$ much less than the cut-off is universal, and is directly related to the Rényi entropy. This because its Laplace transform is given by correlation functions of twist operators in imaginary time:

$$
\begin{aligned}
\int P(E) e^{-E \tau} d E & ={ }_{H}\left\langle 0\left|e^{-\left(H^{\prime}-H\right) \tau}\right| 0\right\rangle_{H} \\
& ={ }_{H}\langle 0|\left(\prod_{k} \mathcal{T}_{n}\left(l_{k}, \tau\right)^{\dagger}\right)\left(\prod_{k} \mathcal{T}_{n}\left(l_{k}, 0\right)|0\rangle_{H}\right.
\end{aligned}
$$

We now consider various simple cases of this.

Single twist in an infinite ungapped system. At the critical point the two-point function $\left\langle\mathcal{T}_{n}(0, \tau)^{\dagger} \mathcal{T}_{n}(0,0)\right\rangle \sim$ $b_{n} \tau^{-2 x_{n}}$, where $b_{n}$ is the same constant appearing in the result for the Rényi entropy of an interval of length $\ell$ (in units where $v=1$.) In this case the spectrum of $H^{\prime}$ is continuous and we see that $P(E) d E \sim$ $\left(b_{n} / \Gamma\left(2 x_{n}\right)\right) E^{2 x_{n}-1} d E$. Note that the probability of finding the system in a state of energy $<E$ approaches zero as $E \rightarrow 0$. This is an example of an 'orthogonality catastrophe' as was first observed in the X-ray edge singularity [26]. As in that problem, the probability of occupation of low-lying states obeys a universal power law [27].

Single twist in a gapped system. When the theory is gapped, the 2-point function behaves asymptotically like $\left|\left\langle\mathcal{T}_{n}\right\rangle\right|^{2}$, giving a probability $P_{0} \sim b_{n}^{\prime} \Delta^{2 x_{n}}$ that the system is in the ground state. More interesting is the occupation of low lying states. In 28] it was shown that $\mathcal{T}_{n}$ couples to multi-particle states in the continuum. As an example, the coupling to 2-particle states with $E>2 \Delta$ gives

$$
P(E)=P_{0} \sum_{k_{1}, k_{2}} \sum_{i=1}^{n} \sum_{j=1}^{n}\left|F_{i j}\left(k_{1}, k_{2}\right)\right|^{2} \delta\left(E-E_{k_{1}}-E_{k_{2}}\right)
$$

where $F_{i j}$ is a form factor coupling $\mathcal{T}_{n}$ to a particle of momentum $k_{1}$ in copy $i$ and of momentum $k_{2}$ in copy $j$. For integrable models these are calculable [28] and thus measurement of $P(E)$ gives direct access to $P_{0}$ and therefore the Rényi entropy.

Single twist in a finite ungapped system. Suppose the initial system has finite length $L$ with open boundary conditions. The form of the 2-point function 
$\left\langle\mathcal{T}_{n}(x, \tau)^{\dagger} \mathcal{T}_{n}(x, 0)\right\rangle$ can be found by a conformal mapping to the upper half plane. As $\tau \rightarrow \infty$ we find $P_{0}=b_{n} g_{n}((L / \pi) \sin (\pi x / L))^{-2 x_{n}}$, where $x$ is the distance of the twist from one end of the system, and $\log g_{n}$ gives the boundary entropy. This agrees with the result in [4] for the Rényi entropy. The coupling to the excited states can be found using the methods described in [6]. The leading corrections as $\tau \rightarrow \infty$ come from two excitations each of energy $\pi x_{s} / L$ (where $x_{s}$ is a boundary scaling dimension) propagating in two different copies of the available $n$. We then find that

$$
P\left(E=2 \pi x_{s} / L\right)=P_{0} d_{n}\left(x_{s}\right)((L / \pi) \sin (\pi x / L))^{4 x_{b}}
$$

where $d_{n}\left(x_{s}\right)$ is a known function of $x_{s}[6]$.

Multiple twists. This is more complicated still, since for $p$ twists we need to know the $2 p$-point function of twist fields. However analytic results are available from CFT for various limiting cases. For example take the case $p=2$, that is an interval $A$ of length $\ell$ in an infinite system. As discussed above, the probability $P_{0}$ of finding the system in its ground state gives the Rényi entropy. For $\ell \ll \tau$, that is energies $E \ll \ell^{-1}$, the methods of [6] show that the product of two twist operators can be written as an infinite sum over all possible local scaling fields of the CFT on each of the $n$ copies. The leading correction comes from when two of these have dimension $x \neq 0$ and all the rest correspond to the identity. Thus we have

$$
P(E) d E=P_{0} \sum_{x} \tilde{d}_{n}(x)(\ell E)^{4 x-1} d E+\cdots
$$

where $\tilde{d}_{n}(x)$ is once again known, the sum is over all the bulk scaling dimensions, and the neglected terms, of order $(\ell E)^{2\left(x_{i}+x_{j}+x_{k}\right)}$ are also calculable and, for $n>2$, encode further CFT data. (For $n=2$ a closed form for $P(E)$ is available since the 4-pt function of twist operators is related to the partition function of the CFT on a torus [6].) For $E \gg \ell^{-1}$ we find the result for single, independent twists.

Another important example of a local quench, however not related to the Rényi entropy, is where two pieces of a system are joined together to form a larger one. The real time evolution after such a quench was studied in [16, 17]. More recently the fidelity was evaluated using CFT methods [19]. We can also use evolution in imaginary time to predict the population of low energy states following such a quench. In this case, instead of a twist operator, we have a slit in the world sheet, whose scaling dimension in a CFT was computed in [29] to be $c / 16$. Thus, in an infinite system, we have $P(E) d E \propto E^{(c / 8)-1} d E$.

Although the purpose of this article has been to show only that it is possible in principle to measure Rényi entropies, it is interesting to consider whether this proposal is at all practical. As mentioned earlier, imperfections in the faithfulness of the copies or of the precise details of the quench should be unimportant as long as they occur at energy scales much less than the gap $\Delta$ or the energy $E$ above the ground state. In principle, $P(E)$ can be measured if the system couples weakly, in a known manner, to other degrees of freedom whose energy distribution can be spectrally analyzed. For example, if the excited states of $H^{\prime}$ can decay through a coupling to the electromagnetic field, $P(E)$ should be recoverable from the photon spectrum. In optical lattices, the energy $E$ could be transferred to kinetic energy of the atoms, whose spectrum could then be analyzed after removal of the trap. In both cases, in order to produce an observable spectrum, it would of course be necessary to consider a small but finite density of twists, produced continuously at a low rate. Under such conditions, the above analysis shows that we should obtain simple universal behavior, characteristic of independent twists, for energies $E$ larger than mean density (in units where $v=1$ ), but much smaller than the band width.

Although we have restricted attention to case of one dimension, where twists are local, the analysis in principle extends to higher dimensions. In particular it can be seen that the action of twist operators can change the topology and so reveal the entanglement entropy of topological phases. We have throughout assumed that the ground state $|0\rangle$ is unique, and there are interesting consequences, even in one dimension, when this is not the case.

The author thanks P. Calabrese, B. Doyon, F. Essler, P. Fendley, E. Fradkin and A. Silva for useful comments, and the KITP, Santa Barbara, for its hospitality. This research was supported in part by the EPSRC under Grant EP/D050952/1 and by the National Science Foundation under Grant NSF PHY05-51164.

[1] M. Srednicki, Phys. Rev. Lett. 71, 666 (1993).

[2] For a review, see P. Calabrese and J. Cardy, J. Phys. A 42, 504005 (2009).

[3] C. Holzhey, F. Larsen, and F. Wilczek, Nucl. Phys. B 424, 44 (1994); C. Callan and F. Wilczek, Phys. Lett. B 333, 55 (1994).

[4] P. Calabrese and J. Cardy, J. Stat. Mech., 0406:P06002 (2004).

[5] P. Calabrese, J. Cardy and E. Tonni, J. Stat. Mech., P11001 (2009).

[6] P. Calabrese, J. Cardy and E. Tonni, J. Stat. Mech. P01021, (2011).

[7] J.I. Cirac and F. Verstraete, J. Phys. A: Math. Theor. 42, 504004 (2009).

[8] P. Horodecki and A. Ekert, Phys. Rev. Lett. 89, 127902 (2002).

[9] I. Peschel, J. Phys. A 36, L205 (2003).

[10] J.A. Hoyos, A.P. Vieira, N. Laflorencie and E. Miranda, Phys. Rev. B 76, 174425 (2007).

[11] I. Klich, G. Refael and A. Silva, Phys. Rev. A 74, 032306 (2006). 
[12] I. Klich and L. Levitov, Phys. Rev. Lett. 102, 100502 (2009).

[13] H.F. Song, C. Flindt, S. Rachel, I. Klich, and K. Le Hur, arXiv:1008.5191.

[14] H.F. Song, S. Rachel, and K. Le Hur, arXiv:1002.0825.

[15] B. Hsu, E. Grosfeld and E. Fradkin, Phys. Rev. B 80, 235412 (2009).

[16] V. Eisler and I. Peschel, J. Stat. Mech. P06005 (2007).

[17] P. Calabrese and J. Cardy, J. Stat. Mech. P10004 (2007).

[18] L. Campos Venuti and P. Zanardi, Phys. Rev. Lett. 99, 096701 (2007).

[19] J. Dubail and J.-M. Stéphan, arXiv:1010.3716.

[20] C. De Grandi, V. Gritsev and A. Polkovnikov, Phys. Rev. B 81, 012303 (2009).

[21] A. Silva, Phys. Rev. Lett. 101, 120603 (2008).

[22] M.B. Hastings, I. Gonzalez, A.B. Kallin and R.G. Melko,
Phys. Rev. Lett. 104, 157201 (2010).

[23] P. Zanardi, C. Zolka and L. Faoro, Phys. Rev. A 62, 030301 (2000).

[24] O.A. Castro-Alvaredo and B. Doyon, J.Stat.Mech. 1102:P02001, (2011).

[25] L.J. Dixon, D. Friedan, E.J. Martinec and S.H. Shenker, Nucl. Phys. B 282, 13 (1987).

[26] P.W. Anderson, Phys. Rev. Lett. 18, 1049 (1967).

[27] K.D. Schotte KD and U. Schotte, Phys. Rev. 182, 479 (1969); I. Affleck and A.W.W Ludwig, J. Phys. A: Math. Gen. 27, 5375 (1994).

[28] J.L. Cardy, O.A. Castro-Alvaredo and B. Doyon, J. Stat. Phys. 130, 129 (2007); O.A. Castro-Alvaredo and B. Doyon, J. Phys. A 42, 504006 (2009).

[29] J. Cardy and I. Peschel, Nucl. Phys. B 300, 377, (1988). 